Research Paper

\title{
Mitochondrial NADH Dehydrogenase Subunit 3 (MTND3) Polymorphisms are Associated with Gastric Cancer Susceptibility
}

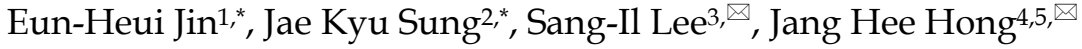 \\ 1. Research Institute for Medical Sciences, Chungnam National University College of Medicine, Daejeon, Republic of Korea \\ 2. Department of Internal Medicine, Chungnam National University Hospital, Chungnam National University College of Medicine, Daejeon, Republic of \\ Korea \\ 3. Department of Surgery, Chungnam National University Hospital, Chungnam National University College of Medicine, Daejeon, Republic of Korea \\ 4. Clinical Trials Center, Chungnam National University Hospital, Daejeon, Republic of Korea \\ 5. Department of Pharmacology, Chungnam National University College of Medicine, Daejeon, Republic of Korea. \\ ${ }^{*}$ Contributed equally to this work.
}

$\square$ Corresponding authors: Sang-Il Lee, M.D., Ph. D., Associate Professor, Department of Surgery, Chungnam National University Hospital, Chungnam National University School of Medicine, 282 Munhwa-ro, Jung-gu, Daejeon, 35015, Republic of Korea. Tel: +82 42 280 7180; Fax: +82 42 257 8024; Email: mr231@cnuh.co.kr. Jang Hee Hong, M.D., Ph. D., Professor, Department of Pharmacology, Chungnam National University College of Medicine, 282 Munhwa-ro, Jung-gu, Daejeon, 35015, Republic of Korea. Tel: +82 42280 6940; Fax: +82 42280 6947; Email: boniii@cnu.ac.kr.

(c) Ivyspring International Publisher. This is an open access article distributed under the terms of the Creative Commons Attribution (CC BY-NC) license (https://creativecommons.org/licenses/by-nc/4.0/). See http://ivyspring.com/terms for full terms and conditions.

Received: 2018.04.25; Accepted: 2018.08.01; Published: 2018.08.10

\begin{abstract}
Accumulating evidence indicates that mitochondrial DNA alterations contribute to cancer development and progression. In this study, we evaluated the relationship between polymorphisms of mitochondrial NADH dehydrogenase subunit 3 (MTND3) and the risk of gastric cancer (GC). Five single nucleotide polymorphisms (SNPs; rs28358278, rs2853826, rs201397417, rs41467651, and rs28358275) were identified and genotyped in 377 patients with GC patients and 363 controls by direct sequencing. The rs $41467651 \mathrm{~T}$ allele was significantly associated with $G C$ risk [adjusted odds ratio $(O R)=2.11,95 \%$ confidence interval $(\mathrm{Cl})=1.25-3.55, P=0.005)$. In stratified analysis, rs28358278, rs2853826, and rs41467651 were associated with subgroups of GC, with the rs28358278 G, rs2853826 T, and rs41467651 T alleles associated with an increased GC risk in females (adjusted $\mathrm{OR}=1.70,95 \% \mathrm{Cl}=1.08-2.69, P=0.023$; adjusted $\mathrm{OR}=1.78,95 \% \mathrm{Cl}=1.11-2.85, P=0.016$; adjusted $O R=2.07,95 \% \mathrm{Cl}=1.04-4.12, P=0.038$, respectively). The rs $441467651 \mathrm{~T}$ allele was also related with GC risk in diffuse-type subjects compared to that of controls (adjusted OR $=2.61,95 \% \mathrm{Cl}=1.43-4.89, P$ $=0.002$ ). In addition, The rs441467651 T allele was significantly related with increased GC risk regardless of lymph node metastasis (LNM), T classification, and tumor stage compared to that of controls (adjusted OR = $2.00,95 \% \mathrm{Cl}=1.12-3.55, P=0.019$ in LNM-negative subjects; adjusted $\mathrm{OR}=2.10,95 \% \mathrm{Cl}=1.05-4.22, P=$ 0.0379 in LNM-positive subjects; adjusted $\mathrm{OR}=1.82,95 \% \mathrm{Cl}=1.02-3.24, P=0.042$ in T1/T2 subjects; adjusted $\mathrm{OR}=2.60,95 \% \mathrm{Cl}=1.29-5.24, P=0.007$ in T3/T4 subjects; adjusted $\mathrm{OR}=1.91,95 \% \mathrm{Cl}=1.09-3.34, P=0.025$ in tumor stage I $(A+B) / I I(A+B+C)$ subjects; adjusted $O R=2.36,95 \% C l=1.12-5.13, P=0.025$ in tumor stage III $(A+B+C)$ subjects) compared to that of controls. Our findings suggest that the rs $28358278, r s 2853826$, and rs41467651 polymorphisms of MTND3 increase the susceptibility to GC development.
\end{abstract}

Key words: Mitochondria, MTND3, polymorphism, gastric cancer

\section{Introduction}

Gastric cancer (GC) is one of the most common cancers diagnosed, and is the third leading cause of cancer-related mortality worldwide [1]. Although the incidence and mortality rates of GC have steadily declined in several countries over the past few decades, these rates continue to be high in Asian countries. In particular, the Korea National Cancer
Center reported that GC was the third-most common cancer in Korea in 2017, with 26,350 new cases and 7,480 deaths recorded $[2,3]$.

Mitochondria play a crucial role in cell energy production in the form of ATP through respiration and oxidative phosphorylation (OXPHO), generation of reactive oxygen species (ROS), and apoptosis $[4,5]$. 
ROS are cytotoxic by-products of the mitochondria that exert damage to both genomic DNA and mitochondrial DNA (mtDNA), which results in tumorigenesis [6, 7]. Indeed, several studies have indicated that mtDNA alterations contribute to the development and progression of various types of cancer, including GC [8-12]. Complex I is the first step in the electron transport system of mitochondrial $\mathrm{OXPHO}$, which is located within the mitochondrial inner membrane, accepts electrons from $\mathrm{NADH}$, and transfers the electrons to ubiquinone. Mitochondrial NADH dehydrogenase subunit 3 (MTND3) is one of seven mtDNA-encoded complex I genes (MTND1, MTND2, MTND3, MTND4, MTND5, MTND6, and MTND7) [13]. Hung et al. [12] identified eight somatic mutations in mtDNA genes, including MTND1, MTND2, MTND5, and D-loop regions, associated with GC. In addition, Bhat et al. [14] reported that the single nucleotide polymorphism at locus rs2853826 in MTND3 increased ROS production in type 2 diabetes mellitus (T2DM). To date, previous studies have shown a significant correlation of polymorphisms in MTND3 with the risk of Parkinson's disease, T2DM, and breast and esophageal cancers, but not with GC $[12,14-19]$. Therefore, the overall association between MTND3 polymorphisms and GC risk remains unknown.

Accordingly, we examined whether MTND3 polymorphisms contribute to GC risk. We first identified SNPs in MTND3 by Sanger sequencing, and then determined their association with an increased risk of GC using a case-control study design. Further, we explored the impact of MTND3 polymorphisms in GC on various characteristics and clinical features, including age, sex, histologic type, lymph node metastasis (LNM), T classification, and tumor stage. These findings should help to clarify population-specific markers of GC and provide insight into uncovering the tumorigenesis mechanisms toward improving the diagnosis and prognosis for patients.

\section{Materials and methods}

\section{Subjects and DNA extraction}

In total, 377 GC patients and 363 healthy controls were enrolled in this study. The blood samples used in this study were provided by the Chungnam National Hospital Biobank, a member of the National Biobank of Korea, which is supported and audited by the Ministry of Health and Welfare of Korea. All individuals enrolled in this study provided their written informed consent for blood collection and use. The study protocol was approved by the Institutional Review Board of the Chungnam National University
Hospital. GC patients were recruited from the outpatient clinic at the Chungnam National University Hospital, and classified according to the Lauren's classification [20]. The controls subjects were randomly selected among healthy volunteers visiting the Chungnam National University Hospital medical center for their annual physical examinations, and included only individuals who had no history of cancer. Genomic DNA was extracted from the peripheral blood using the QIAamp DNA Blood Mini Kit (Qiagen $\mathrm{GmbH}$, Hilden, Germany), according to the manufacturer's instructions.

\section{SNP identification and genotyping}

Polymerase chain reaction (PCR) amplification and Sanger sequencing were performed to identify SNPs and carry out genotyping. PCR primers were designed using the online Primer 3.0 software (http://primer3.ut.ee). The forward primer was 5'-CCACAACTCAACGGCTACAT-3', and the reverse primer was 5'-TGGGTGTTGAGGGTTATGAG-3'. PCR products (491 bp) were sequenced using the same PCR primers and BigDye(R) Terminator v3.1 Cycle Sequencing Kit (Applied Biosytems, Foster City, CA, USA) on an ABI PRISM 3730XL system (Applied Biosystems). The SNPs of MTND3 were detected by sequence analysis based on the reference sequence of human MT: 10398 (GenBank accession number: NC_012920).

\section{Statistical analysis}

Differences in age and gender between the GC and control groups were calculated by the chi-square test and independent $t$-test. Binary logistic regression was used to estimate the GC risk according to odds ratios (ORs) and 95\% confidence intervals (CIs). The association analysis was adjusted by age and sex, which were included as covariates in the model. All statistical analyses were performed using the SPSS (SPSS Inc., Chicago, IL, USA), version 20.0 for Windows. $P<0.05$ was considered statistically significant.

\section{Results}

\section{Characteristic of the subjects and SNP identification}

Characteristics and clinical features of the study subjects (377 GC patients and 363 controls) are summarized in Table 1 . There was no significant difference in the age distribution between groups $(P=$ 0.061 ), whereas the proportion of male subjects was significantly higher in the GC patients $(P<0.001)$. Of the 377 GC patients, 201 (53.3\%) were classified as intestinal type, $136(36.1 \%)$ as diffuse-type, and 40 $(10.6 \%)$ as mixed-type. For LNM, $263(69.8 \%)$ were in 
negative and $114(30.2 \%)$ in positive. For the $\mathrm{T}$ classification, 224 (59.4\%), 54 (14.3\%), 9 (2.4\%), and 90 (23.9\%) were classified to T1, T2, T3, and T4, respectively. For the tumor stage, $260(69.8 \%), 37$ (9.8 $\%)$, and $80(21.2 \%)$ were classified to stage I, II, and III, respectively. Overall, we identified five SNPs in MTND3 in the cases and controls: rs28358278, rs2853826, rs201397417, rs41467651, and rs28358275.

Table 1. Characteristics and clinical features in gastric cancer and control groups

\begin{tabular}{|c|c|c|c|}
\hline Variables & Gastric cancers & Controls & $P$ \\
\hline & $(\mathrm{n}=377)$ & $(\mathrm{n}=363)$ & \\
\hline Age (yr) (mean \pm SD) & $60.1 \pm 11.7$ & $58.7 \pm 9.1$ & $0.061^{*}$ \\
\hline$<60$ & $171(45.4)$ & $186(51.2)$ & $0.122^{\dagger}$ \\
\hline$\geq 60$ & $206(54.6)$ & $177(48.8)$ & \\
\hline \multicolumn{4}{|l|}{ Gender (\%) } \\
\hline Male & $266(70.6)$ & $120(33.1)$ & $<0.001^{\dagger}$ \\
\hline Female & $111(29.4)$ & $243(66.9)$ & \\
\hline \multicolumn{4}{|l|}{ Histological type (\%) } \\
\hline Intestinal & $201(53.3)$ & & \\
\hline Diffuse & $136(36.1)$ & & \\
\hline Mixed & 40 (10.6) & & \\
\hline \multicolumn{4}{|l|}{ T classification (\%) } \\
\hline $\mathrm{T} 1$ & $224(59.4)$ & & \\
\hline $\mathrm{T} 2$ & $54(14.3)$ & & \\
\hline $\mathrm{T} 3$ & $9(2.4)$ & & \\
\hline $\mathrm{T} 4$ & $90(23.9)$ & & \\
\hline \multicolumn{4}{|c|}{ Lymph node metastasis (\%) } \\
\hline Negative & $263(69.8)$ & & \\
\hline Positive & $114(30.2)$ & & \\
\hline \multicolumn{4}{|l|}{ Tumor stage (\%) } \\
\hline $\mathrm{I}(\mathrm{A}+\mathrm{B})$ & $260(70.0)$ & & \\
\hline II $(A+B)$ & $37(9.8)$ & & \\
\hline III $(A+B+C)$ & $80(21.2)$ & & \\
\hline
\end{tabular}

$\mathrm{SD}$, standard deviation.

* Independent $t$-test.

† Two-sided chi-square test.

\section{Associations of SNPs and gastric cancer risk}

To determine whether the rs28358278, rs2853826, rs201397417, rs41467651, and rs28358275 variations of MTND3 were related with the risk of GC, we compared the genotypes frequencies between the case and control groups. The genotype distributions for 5SNPs in MTND3 were shown in Table 2. All ORs and were 95\% CIs adjusted for age and sex for allele and genotype frequencies. The rs41467651 $\mathrm{T}$ allele was significantly associated with GC risk (adjusted OR = 2.11, $95 \% \mathrm{CI}=1.25-3.55, P=0.005)$, whereas the remaining SNPs showed no significant association.

\section{Stratified analysis for five SNPs}

To further evaluate the possible correlation of the five SNPs with GC risk in subgroups, we performed stratified analyses based on various characteristics including age, sex, histologic type, LNM, T classification, and tumor stage. As shown in Table 3, the rs441467651 $\mathrm{T}$ allele was again found to be significantly associated with increased GC risk compared to those of controls in subjects over 60 years old (adjusted OR $=2.40,95 \% \mathrm{CI}=1.14-5.05, P=$ 0.021 ). Moreover, the rs $28358278 \mathrm{G}, \mathrm{rs} 2853826 \mathrm{~T}$, and rs41467651 T allele were associated with increased GC risk compared to those of controls in female subjects (adjusted $\mathrm{OR}=1.70,95 \% \mathrm{CI}=1.08-2.69, P=0.023$; adjusted $\mathrm{OR}=1.78,95 \% \mathrm{CI}=1.11-2.85, P=0.016$; adjusted $\mathrm{OR}=2.07,95 \% \mathrm{CI}=1.04-4.12, P=0.038$, respectively). A significant association was also observed between the rs441467651 $\mathrm{T}$ allele frequency and GC risk in subjects with diffuse-type tumors compared to that of controls (adjusted OR $=2.61,95 \%$ $\mathrm{CI}=1.43-4.89, P=0.002$ ). However, the rs441467651 T allele was significantly related with increased GC risk regardless of LNM, T classification, or tumor stage compared to that of controls (adjusted OR $=2.00,95 \%$ $\mathrm{CI}=1.12-3.55, P=0.019$ in LNM negative subjects; adjusted $\mathrm{OR}=2.10,95 \% \mathrm{CI}=1.05-4.22, P=0.0379$ in LNM positive subjects; adjusted $\mathrm{OR}=1.82,95 \% \mathrm{CI}=$ 1.02-3.24, $P=0.042$ in T1/T2 subjects; adjusted OR = 2.60, $95 \% \mathrm{CI}=1.29-5.24, P=0.007$ in T3/T4 subjects; adjusted $\mathrm{OR}=1.91,95 \% \mathrm{CI}=1.09-3.34, P=0.025$ in tumor stage $\mathrm{I}(\mathrm{A}+\mathrm{B}) / \mathrm{II}(\mathrm{A}+\mathrm{B}+\mathrm{C})$ subjects; adjusted $\mathrm{OR}$ $=2.36,95 \% \mathrm{CI}=1.12-5.13, P=0.025$ in tumor stage III $(\mathrm{A}+\mathrm{B}+\mathrm{C})$ subjects) compared to that of controls.

Table 2. Genotypes/alleles frequency of DN3 polymorphisms between gastric cancers and controls as well as the association with risk of GC

\begin{tabular}{|c|c|c|c|c|c|c|c|}
\hline SNP & Contig position & Protein position & Genotype & GC, N (\%) & CON, N (\%) & OR $(95 \% \mathrm{CI})^{*}$ & $P$ \\
\hline \multirow[t]{2}{*}{ rs28358278 } & 10400 & 114 & A & $222(58.9)$ & $228(62.8)$ & 1.00 (ref.) & \\
\hline & & & G & 155 (41.1) & 135 (37.2) & $1.23(0.90-1.71)$ & 0.190 \\
\hline \multirow[t]{2}{*}{ rs2853826 } & 10398 & 114 & C & $244(64.7)$ & $248(68.3)$ & 1.00 (ref.) & \\
\hline & & & $\mathrm{T}$ & $133(35.3)$ & $115(31.7)$ & $1.20(0.86-1.67)$ & 0.300 \\
\hline \multirow[t]{2}{*}{ rs201397417 } & 10345 & 96 & A & 361 (95.8) & 355 (97.8) & 1.00 (ref.) & \\
\hline & & & G & $16(4.2)$ & $8(2.2)$ & $1.43(0.57-3.58)$ & 0.441 \\
\hline \multirow[t]{2}{*}{ rs41467651 } & 10310 & 84 & C & $325(86.2)$ & 335 (92.3) & 1.00 (ref.) & \\
\hline & & & $\mathrm{T}$ & $52(13.8)$ & $28(7.7)$ & $2.11(1.25-3.55)$ & 0.005 \\
\hline \multirow[t]{2}{*}{ rs28358275 } & 10237 & 60 & A & $363(96.3)$ & $350(96.4)$ & 1.00 (ref.) & \\
\hline & & & G & $14(3.7)$ & $13(3.6)$ & $0.87(0.38-1.98)$ & 0.732 \\
\hline
\end{tabular}

$\mathrm{SNP}$, single nucleotide polymorphism; GC, gastric cancer; CON, controls; OR, odds ratio; CI, confidence interval; ref, reference.

* Adjusted by age and gender.

The significant results are in bold. 
Table 3. Association between each SNP and clinical characteristics of gastric cancer

\begin{tabular}{|c|c|c|c|c|c|c|c|c|c|c|}
\hline \multirow[t]{3}{*}{ Variables } & \multicolumn{10}{|l|}{ GC vs. CON } \\
\hline & \multicolumn{2}{|l|}{ rs28358278 } & \multicolumn{2}{|l|}{ rs2853826 } & \multicolumn{2}{|l|}{ rs201397417 } & \multicolumn{2}{|l|}{ rs41467651 } & \multicolumn{2}{|l|}{ rs28358275 } \\
\hline & OR $(95 \% \mathrm{CI})^{*}$ & $P$ & OR $(95 \% \mathrm{CI})^{*}$ & $P$ & OR $(95 \% \mathrm{CI})^{*}$ & $P$ & OR $(95 \% \mathrm{CI})^{*}$ & $P$ & OR $(95 \% \mathrm{CI})^{*}$ & $P$ \\
\hline \multicolumn{11}{|l|}{ Age (years) } \\
\hline$<60$ & $1.49(0.91-2.45)$ & 0.113 & $1.47(0.88-2.44)$ & 0.141 & $1.56(0.35-6.94)$ & 0.558 & $1.26(0.57-2.83)$ & 0.569 & $3.14(0.70-14.18)$ & 0.136 \\
\hline$\geq 60$ & $0.94(0.60-1.46)$ & 0.775 & $0.91(0.58-1.44)$ & 0.693 & $1.50(0.42-5.36)$ & 0.536 & $2.40(1.14-5.05)$ & 0.021 & $0.40(0.12-1.38)$ & 0.149 \\
\hline \multicolumn{11}{|l|}{ Sex } \\
\hline Male & $0.91(0.59-1.42)$ & 0.686 & $0.82(0.52-1.29)$ & 0.386 & $1.39(0.44-4.40)$ & 0.578 & $1.96(0.87-4.38)$ & 0.103 & $0.71(0.25-2.02)$ & 0.524 \\
\hline Female & $1.70(1.08-2.69)$ & 0.023 & $1.78(1.11-2.85)$ & 0.016 & $1.10(0.20-6.15)$ & 0.915 & $2.07(1.04-4.12)$ & 0.038 & $0.91(0.23-3.60)$ & 0.892 \\
\hline \multicolumn{11}{|l|}{ Histological type } \\
\hline Intestinal & $1.01(0.68-1.51)$ & 0.947 & $1.03(0.69-1.55)$ & 0.787 & $0.62(0.22-1.74)$ & 0.361 & $1.81(0.95-3.47)$ & 0.073 & $1.40(0.51-3.83)$ & 0.510 \\
\hline Diffuse & $0.76(0.50-1.15)$ & 0.187 & $1.25(0.81-1.91)$ & 0.312 & $1.17(0.29-4.64)$ & 0.826 & $2.61(1.43-4.89)$ & 0.002 & $0.85(0.30-2.37)$ & 0.753 \\
\hline \multicolumn{11}{|c|}{ Lymph node Metastasis } \\
\hline Negative & $1.19(0.83-1.69)$ & 0.346 & $1.18(0.82-1.70)$ & 0.371 & $1.40(0.52-3.78)$ & 0.505 & $2.00(1.12-3.55)$ & 0.019 & $0.94(0.38-2.34)$ & 0.890 \\
\hline Positive & $1.24(0.49-1.94)$ & 0.352 & $1.08(0.68-1.72)$ & 0.750 & $1.05(0.25-4.31)$ & 0.951 & $2.10(1.05-4.22)$ & 0.037 & $0.58(0.16-0.21)$ & 0.411 \\
\hline \multicolumn{11}{|l|}{$\mathrm{T}$ classification } \\
\hline $\mathrm{T} 1 / \mathrm{T} 2$ & $1.17(0.82-1.66)$ & 0.384 & $1.15(0.80-1.65)$ & 0.449 & $1.33(0.49-3.59)$ & 0.573 & $1.82(1.02-3.24)$ & 0.042 & $1.04(0.43-2.53)$ & 0.938 \\
\hline $\mathrm{T} 3 / \mathrm{T} 4$ & $1.30(0.81-2.10)$ & 0.274 & $1.14(0.70-1.86)$ & 0.603 & $1.25(0.30-5.24)$ & 0.757 & $2.60(1.29-5.24)$ & 0.007 & $0.44(0.10-2.07)$ & 0.302 \\
\hline \multicolumn{11}{|l|}{ Tumor stage } \\
\hline $\mathrm{I}(\mathrm{A}+\mathrm{B}) / \mathrm{II}(\mathrm{A}+\mathrm{B}+\mathrm{C})$ & $1.19(0.84-1.67)$ & 0.332 & $1.18(0.83-1.69)$ & 0.350 & $1.23(0.46-3.31)$ & 0.682 & $1.91(1.09-3.34)$ & 0.025 & $0.90(0.37-2.18)$ & 0.808 \\
\hline III $(\mathrm{A}+\mathrm{B}+\mathrm{C})$ & $1.26(0.76-2.12)$ & 0.374 & $1.01(0.59-1.72)$ & 0.985 & $1.64(0.39-6.86)$ & 0.501 & $2.39(1.12-5.13)$ & 0.025 & $0.57(0.12-2.65)$ & 0.472 \\
\hline
\end{tabular}

$\mathrm{GC}$, gastric cancer; $\mathrm{CON}$, controls; $\mathrm{OR}$, odds ratio; $\mathrm{CI}$, confidence interval.

* Adjusted by age and gender.

The significant results are in bold.

\section{Discussion}

To date, several studies, including a meta-analysis, have reported that among the identified MTND3 SNPs, rs2853826 is related to the risk of Parkinson's disease, T2DM, and breast and esophageal cancers, but not to GC $[12,14-19,21]$. In the present study, we scanned Korean-specific polymorphisms of patients with GC and healthy subjects by direct sequencing of the MTND3 gene, and identified five SNPs: rs28358278, rs2853826, rs201397417, rs41467651, and rs28358275. However, we did not detect any Korean-specific novel SNP. The rs28358278 (Thr114Thr) and rs41467651 (Leu84Leu) SNPs are synonymous coding variants, whereas rs2853826 (Thr114Ala), rs201397417 (Ile96Thr), and rs28358275 (Ile60Thr) are non-synonymous variants. Consistent with the HapMap Asian SNP database (phase 3), no heteroplasmic SNPs were found in MTND3 in our study population.

We investigated the possible correlation between the five SNPs and general GC risk as well as among GC subgroups. Overall, we found an association between the SNP rs41467651 and an increased risk of GC. In stratified analysis, rs28358278, rs2853826, and rs41467651 were also associated with an increased risk of GC in women, indicating an interaction of the effects of these SNPs with gender. Moreover, the rs441467651 SNP was associated with a 2.61-times increased risk of GC in patients with diffuse-type tumors than controls, representing the highest OR detected for all associations between the SNPs and the risk of GC in subgroups. Furthermore, a positive association was observed between rs41467651 and increased GC risk regardless of LNM, T classification, or tumor stage. Although the OR of rs41467651 was similar for LNM-negative and -positive patients, it was higher in the T3/T4 GC subgroup than in the T1/T2 GC subgroup. The GC risk (2.39) of harboring the rs41467651 SNP was also higher for patients in the tumor stage III $(A+B+C)$ subgroup than for those in the tumor stage $\mathrm{I}(\mathrm{A}+\mathrm{B}) / \mathrm{II}(\mathrm{A}+\mathrm{B}+\mathrm{C})$ subgroup. These results demonstrated that although the rs41467651 SNP is a synonymous polymorphism, it can nevertheless contribute to the development and progression of GC.

There are some limitations of our study that should be mentioned. First, the sample size was too small to have sufficient statistical power in the stratified analysis. Second, although the role of Helicobacter pylori infection as a GC risk factor is controversial, we did not investigate the relevance of MTND3 polymorphism in relation to $H$. pylori in GC owing to the unavailability of such data. Finally, we did not explore potential associations between smoking, drinking, and diet to the relationship of GC risk and polymorphisms owing to the unavailability of these data for our cohort. Thus, the effects of these factors on mediating GC risk will be assessed in future studies.

Despite these limitations, our study is the first to explore the association between MTND3 SNPs and risk of GC and GC subgroups. Although the sample size was too small for stratified analysis, our findings suggest that the rs28358278, rs2853826, and rs41467651 SNPs in MTND3 might increase susceptibility to developing GC. However, further detailed study of the function and mechanism are needed to clarify the impact of these SNPs in MTND3. 


\section{Abbreviations}

MTND3, mitochondrial NADH dehydrogenase subunit 3; GC, gastric cancer; SNP, single nucleotide polymorphisms; LNM, lymph node metastasis; OXPHO, oxidative phosphorylation; ROS, reactive oxygen species; mtDNA, mitochondrial DNA; T2DM, type 2 diabetes mellitus.

\section{Acknowledgements}

This research was supported by Chungnam National University research fund.

\section{Competing Interests}

The authors have declared that no competing interest exists.

\section{References}

1. Ferlay J, Soerjomataram I, Dikshit R, et al. GLOBOCAN 2012: Estimated cancer incidence mortality, and prevalence worldwide in 2012 v1.0. IARC CancerBase No. 11. Available from http:/ / globocan.iarc.fr (accessed on January 15, 2014).

2. Jemal A, Bray F, Center MM, Ferlay J, Ward E, Forman D. Global cancer statistics. CA Cancer J Clin. 2011; 61: 69-90.

3. Jung KW, Won YJ, Kong HJ, Oh CM, Kong HJ, Lee DH, Lee KH. Prediction of cancer incidence and mortality in Korea, 2017. Cancer Res Treat. 2017; 49: 306-12.

4. Verma M, Kumar D. Application of mitochondrial genome information in cancer epidemiology. Clin Chim Acta. 2007; 383: 41-50.

5. Cavalli LR, Liang BC. Mutagenesis, tumorigenicity, and apoptosis: are the mitochondria involved? Mutat Res. 1998; 398: 19-26.

6. Kang D, Hamasaki N. Mitochondrial oxidative stress and mitochondrial DNA. Clin Chem Lab Med. 2003; 41: 1281-8.

7. Richard SM, Bailliet G, Páez GL, Bianchi MS, Peltomäki P, Bianchi NO. Nuclear and mitochondrial genome instability in human breast cancer. Cancer Res. 2000; 60: 4231-7.

8. Petros JA, Baumann AK, Ruiz-Pesini E, et al. mtDNA mutations increase tumorigenicity in prostate cancer. Proc Natl Acad Sci USA. 2005; 102: 719-24.

9. Zhou S, Kachhap S, Sun W, et al. Frequency and phenotypic implications of mitochondrial DNA mutations in human squamous cell cancers of the head and neck. Proc Natl Acad Sci USA. 2007; 104: 7540-5.

10. Bai RK, Leal SM, Covarrubias D, Liu A, Wong LJ. Mitochondrial genetic background modifies breast cancer risk. Cancer Res. 2007; 67: 4687-94.

11. Wu CW, Yin PH, Hung WY, et al. Mitochondrial DNA mutations and mitochondrial DNA depletion in gastric cancer. Genes Chromosomes Cancer. 2005; 44: 19-28.

12. Chomyn, A., Cleeter, W. J., Ragan, C. I., Riley, M., Doolittle, R. F., Attardi, G. URF6, last unidentified reading frame of human mtDNA, codes for an NADH dehydrogenase subunit. Science 234: 614-618, 1986. Chomyn, A., Cleeter, W. J., Ragan, C. I., Riley, M., Doolittle, R. F., Attardi, G. URF6, last unidentified reading frame of human mtDNA, codes for an NADH dehydrogenase subunit. Science 234: 614-618, 1986.Hung WY, Wu CW, Yin PH, et al. Somatic mutations in mitochondrial genome and their potential roles in the progression of human gastric cancer. Biochim Biophys Acta. 2010; 1800: 264-70.

13. Chomyn A, Cleeter MW, Ragan CI, Riley M, Doolittle RF, Attardi G. URF6, last unidentified reading frame of human mtDNA, codes for an NADH dehydrogenase subunit. Science. 1986; 234: 614-8.

14. van der Walt JM, Nicodemus KK, Martin ER, et al. Mitochondrial polymorphisms significantly reduce the risk of Parkinson disease. Am J Hum Genet. 2003; 72: 804-811.

15. Bhat A, Koul A, Sharma S, et al. The possible role of 10398A and 16189C mtDNA variants in providing susceptibility to T2DM in two North Indian populations: a replicative study. Hum Genet. 2007; 120: 821-6.

16. van der Walt JM, Nicodemus KK, Martin ER, et al. Mitochondrial polymorphisms significantly reduce the risk of Parkinson disease. Am J Hum Genet. 2003; 72: 804-11.

17. Bai RK, Leal SM, Covarrubias D, Liu A, Wong LJ. Mitochondrial genetic background modifies breast cancer risk. Cancer Res. 2007; 67: 4687-94.

18. Darvishi K, Sharma S, Bhat AK, Rai E, Bamezai RN. Mitochondrial DNA G10398A polymorphism imparts maternal haplogroup $\mathrm{N}$ a risk for breast and esophageal cancer. Cancer Lett. 2007; 249: 249-55.

19. Pezzotti A, Kraft P, Hankinson SE, Hunter DJ, Buring J, Cox DG. The mitochondrial A10398G polymorphism, interaction with alcohol consumption, and breast cancer risk. PLoS One. 2009; 4: e5356.
20. Grzybowska-Szatkowska L, Slaska B. Mitochondrial NADH dehydrogenase polymorphisms are associated with breast cancer in Poland. J Appl Genet. 2014; 55: 173-81.

21. Lauren P. The two histological main types of gastric carcinoma: diffuse and so-called intestinal-type carcinoma. an attempt at a histo-clinical classification. Acta Pathol Microbiol Scand. 1965; 64: 31-49.

22. Mao Q, Gao L, Liu Q, et al. The A10389G polymorphism of ND3 gene and breast cancer: a meta-analysis. Biomed Rep. 2013; 1: 259-64. 